\title{
Positivity Rate of Pyrosequencing to Diagnose Drug-Resistant Tuberculosis Directly from Sputum with Different Bacterial Load
}

\author{
Selma Zein Syafira ${ }^{1}$, Nabilla Ghina Zavitri², $\mathrm{Su} \mathrm{Yan}^{3}$, Yunia Sribudiani ${ }^{4}$, \\ Alexander Lezhava ${ }^{3}$, Lidya Chaidir ${ }^{4, *}$ \\ ${ }^{1}$ Faculty of Medicine, Universitas Padjadjaran, Jl. Eijkman No. 38, Bandung 40161, Indonesia \\ ${ }^{2}$ Infectious Disease Research Center, Faculty of Medicine, Universitas Padjadjaran, Jl. Eijkman No. 38, Bandung 40161, Indonesia \\ ${ }^{3}$ Genome Institute of Singapore, 60 Biopolis St, Singapore 138672, Singapore \\ ${ }^{4}$ Department of Biomedical Sciences, Faculty of Medicine, Universitas Padjadjaran, Jl. Eijkman No. 38, Bandung 40161, Indonesia \\ ${ }^{*}$ Corresponding author. E-mail: lidya.chaidir@unpad.ac.id
}

Received date: Jan 10, 2020; Revised date: Aug 22, 2020; Accepted date: Sep 1, 2020

\section{Abstract}

$\mathrm{B}$

ACKGROUND: Molecular techniques, which detect mutations associated with drug resistance tuberculosis (TB), are promising technologies for rapid diagnosis and monitoring of drug-resistant TB. Pyrosequencing is a potential rapid and robust molecular technique to detect drug resistance but its performance in clinical samples is less investigated. This study aimed to determine the positivity rate of pyrosequencing to diagnose drug-resistant TB directly from sputum samples with different grades of sputum smear microscopy results.

METHODS: Thirty-five sputum specimens from drugresistant TB suspects were submitted for acid-fast bacilli (AFB) microscopy. All specimens were cultured using microscopic observation drug susceptibility (MODS) culture. Pyrosequencing was performed to DNA extracted from sputum of culture-positive patients.

RESULTS: MODS culture was positive in 19/35 subjects (54.29\%) samples; 16 smear-positive and three smear-negative. Using pyrosequencing, Mycobacterium tuberculosis was identified in all culture-positive samples, including smear-negative samples. A complete resistance profile for $16(82.35 \%)$ samples could be generated. Pyrosequencing failed to show results for eis or gyr promoter in three samples. Nine of 19 patients were multidrug resistant-TB (MDR-TB), 1/19 was rifampicinresistance TB (RR-TB), and 4/19 were pre-extensively drugresistant TB (pre-XDR-TB). Two novel mutations in $r p o B$ and $r r s$ (associated with rifampicin and aminoglycoside, respectively) were found in this study.

CONCLUSION: The results of this study demonstrates high positivity rates of pyrosequencing to detect drug-resistant TB directly from sputum samples with different grades of smear microscopy, as the surrogate of bacterial load. The assay can be used as a first prediction test of drug resistance prior to confirmation by phenotypic tests.

KEYWORDS: drug-resistant tuberculosis, pyrosequencing, direct sputum

Indones Biomed J. 2020; 12(4): 313-9

\section{Introduction}

Tuberculosis (TB) is a leading cause of death from infectious disease caused by Mycobacterium tuberculosis bacteria.(1) The reatment remains a major challenge because M. tuberculosis cell wall characteristics that are thick and layered, and its ability to make new mutations that will lead to further resistance.(2) World Health Organization (WHO) estimates about half a million cases of multidrug resistantTB (MDR-TB), defined as the resistance of M. tuberculosis against at least rifampicin and isoniazid, emerged globally. The numbers increased between 2017-2018 in several high burden countries, including Indonesia.(3)

The diagnosis of drug-resistant TB requires drug susceptibility testing to determine $M$. tuberculosis resistance 
to certain antituberculosis drugs. Culture-based tests are considered as the gold standard for diagnosing drug-resistant $\mathrm{TB}$, but it can take weeks to months to obtain results, make it less useful for treatment decisions.(4) Long diagnostic turnaround time and the use of incorrect and inappropriate drugs can be detrimental because they can contribute to the amplification of $M$. tuberculosis resistance patterns to antituberculosis drugs. $(5,6)$

Diagnostic time of drug-resistant $\mathrm{TB}$ has been dramatically reduced in recent years by worldwide implementation of molecular tests, which enable the prediction of resistance based on resistance-conferring mutations.(4) One of the methods is Xpert MTB/Rif which can detect $M$. tuberculosis and its resistance to rifampicin simultaneously. Several studies reported that Xpert MTB/ Rif has high sensitivity (92-93\%) and specificity (9398\%) against $M$. tuberculosis culture. but it can only detect resistance to rifampicin. $(7,8)$ Culture-based tests are still needed to predict resistance profiles of other antituberculosis drugs. Another rapid molecular test, line probe assay (LPA), can detect more resistance including isoniazid, rifampicin, fluoroquinolone, and second-line injectable drugs. This assay has a good accuracy for smear-positive specimens and M. tuberculosis culture.(9) However, most commercially available tests are restricted to few drug targets only and mostly rely on the detection of predetermined mutations, so bacteria with novel drug resistance mutations are frequently missed.(10) Several studies reported that the presence of novel mutations are associated with a variable level of particular drugs resistance.(11-13)

More recently, gene sequencing such as pyrosequencing (PSQ) has become a more effective method for rapid detection of drug-resistant $\mathrm{TB}$ in pulmonary samples. PSQ is rapid and provides information about all potential resistance-conferring mutations, including novel mutations, in particular genes with high throughput multilocus capacity.(14) The accuracy of PSQ is comparable to other methods of gene sequencing. $(10,15)$ The current PSQ platform uses culture isolates, prepending additional time for the samples to be sequenced.(16) The performance of this method to predict drug-resistant TB directly from sputum samples is largely unknown.

In this study, we aim to determine the positivity rate of PSQ to detect drug-resistant TB directly from sputum samples with varied grades of smear positivity according to International Union Against Tuberculosis and Lung Diseases (IUATLD) grading scales. M. tuberculosis culture was used as a gold standard. We expect that PSQ platform presented in this study can reduce the time for diagnosis of drug-resistant TB and reduce empirical antibiotic therapy, thereby reducing patient's exposure to unnecessary medications.

\section{Methods}

\section{Sample Collection}

This was an experimental laboratory study using sputum specimens collected consecutively from patients suspected to have drug-resistant TB. The study was conducted from August to December 2019. A total of 55 specimens were collected consecutively from the MDR-TB clinic of Dr. Hasan Sadikin General Hospital, Bandung, Indonesia. All samples were taken from adult patients $(>18$ years old) who met the criteria of drug-resistant TB suspect according to the Indonesian Ministry of Health.(17) The criteria include TB patients with treatment failure, relapse, defaulter, or subjects who had close contact with drug-resistant TB patients. Twenty samples were excluded because the volume was less than $0.5 \mathrm{~mL}$ and/or contained more saliva. The study was approved by the Ethics Committee of Faculty of Medicine, Universitas Padjadjaran, Bandung (number 1109/ UN6.KEP/EC/2019).

\section{Sputum Smear Microscopy}

Acid-fast bacilli (AFB) smear examination was performed using the Ziehl-Neelsen staining technique to detect the presence of Mycobacteria. Sputum was smeared on a glass slide and stained according to a standardized protocol. The stained slides were observed under a light microscope with 1000x magnification. The results were graded and reported according to the IUATLD grading scale as negative, scanty, $1+, 2+$, or $3+$ based on the demonstration of AFB under light microscopy.

\section{Sputum Culture and Phenotypic Drug Susceptibility Testing}

After smear examination, sputum samples were decontaminated with sodium hydroxide-N-AcetylL-Cysteine (NaOH-NALC) method. One milliliter of decontaminated samples was used for microscopic observation of drug susceptibility (MODS) culture as a gold standard in this study, and an amount of $200 \mathrm{uL}$ decontaminated sputum was stored for DNA extraction for PSQ.

MODS culture used liquid-based media Middlebrook 7H9 enriched with oleic acid, albumin, dextrose, and catalase (OADC). MODS culture was performed as described in the 
MODS-user guide.18 Briefly, 5.9 g Middlebrook 7H9 broth (Difco, Sparks, MD, USA), $3.1 \mathrm{~mL}$ glycerol, and $1.25 \mathrm{~g}$ bacto casitone (Difco) in $880 \mathrm{~mL}$ sterile distilled water were prepared to make MODS media. Each day, one MODS plate with the addition of $2.5 \mathrm{~mL}$ OADC (Becton Dickinson, Franklin Lakes, NJ, USA) and $500 \mu \mathrm{L}$ PANTA antibiotic (Becton Dickinson) was set up. Nine-hundred $\mu \mathrm{L}$ medium was aliquoted to each well with $100 \mu \mathrm{L}$ decontaminated sputum. H37Rv as positive control and sterile distilled water as negative control well were also inoculated each day. Phenotypic drug susceptibility testing was performed at a concentration of $40 \mathrm{ug} / \mathrm{mL}$ for rifampicin and $0.2 \mathrm{ug} / \mathrm{mL}$ for isoniazid. The 24 -well plate was then inserted into a sealed polyethylene plastic and incubated at $37^{\circ} \mathrm{C}$ temperature.

The plate was observed for the evidence of $M$. tuberculosis growth on the fourth day of the incubation and was continued on day $5,6,7,8,14,21,28,35$, and 42 . The results of the observation were reported according to the user guide. A negative culture result was reported after 42 days of incubation.

\section{DNA Extraction}

DNA extraction was performed on stored decontaminated sputum samples which showed positive MODS culture. Negative culture samples were not investigated as they did not show the evidence of TB. DNA was extracted using QiaAmp DNA Mini Kit (Qiagen, Hilden, Germany) with a minor modification by adding lysozyme before cell lysis procedure. Preparation of the solutions and the samples were performed according to the manufacturer's instructions (Pyrobett MXDR-TB Test User Manual).

Briefly, decontaminated samples were centrifuged at $17.000 \mathrm{~g}$ for 10 minutes. After discarding the supernatant, lysozyme solution was added, and the mixture was incubated at $37^{\circ} \mathrm{C}$ for one hour. Lysis buffer was added to the mixture and was incubated at $56^{\circ} \mathrm{C}$ temperature for 30 minutes, followed by $95^{\circ} \mathrm{C}$ for 15 minutes, and then cooled down at room temperature. Absolute ethanol was then added, and the mixture was transferred into a $2 \mathrm{~mL}$ spin column (in a $2 \mathrm{~mL}$ collection tube). The spin column was centrifuged at 6.000 $\mathrm{g}$ for 1 minute and placed into a new $2 \mathrm{~mL}$ tube while the previous tube containing filtrate was discarded and washed with AW1 and AW2 buffer. Extracted DNA was used for amplification and PSQ.

\section{Pyrosequencing}

Before PSQ, a real-time PCR was performed for each sample on a Rotor-Gene Q platform (Qiagen) as per the manufacturer's instructions (Pyrobett MXDR-TB Test User
Manual). The sequence 'IS6110' was used as the molecular target for identifying the $M$. tuberculosis complex. The platform requires at least 5 samples per run. The samples and positive controls should produce specific melting curves (Tms), and the PCR products were then analyzed with PSQ for the presence of drug resistance mutations.

PSQ was performed on a PyroMark Q48 Autoprep platform (Qiagen) equipped with Qseq software to identify sequences of the samples. The sequencing primers (sequences as requested) and the nucleotide dispensation order used were described in the manufacturer's instructions. This study determined eight genes: IS6110 for identification of $M$. tuberculosis complex, katG315, and promotor region of inhA for isoniazid resistance, rrs 14011402, and promotor region of eis for aminoglycoside resistance, gyrA quinolone resistance determining region for fluoroquinolone resistance, and two sets of $r p o B$ hotspot region for rifampicin resistance.

The procedures were described within the platform. The preparation consisted of replacing the dry, clean absorber strip into the PSQ platform and filling the injectors containing nucleotides (A, C, T, and $\mathrm{G}$ ), sequencing primers (P1 and P2), and reagents from the PyroMark kit into the cartridge in the order written on the platform. The PyroMark disc was installed into the platform, and the template (PCR products) and magnetic beads were placed into the disc. The pyrogram of each target was analyzed for the presence of mutations associated with drug resistance.

\section{Data Analysis}

The positivity rate of pyrosequencing was expressed as a percentage. Sensitivity and specificity of pyrosequencing in drug susceptibility testing for rifampicin and isoniazid were calculated using MODS as the reference standard. Time to culture positivity was expressed as median days.

\section{Results}

A total of 35 sputum specimens were collected from TB patients with relapse $(22 / 35 ; 62.86 \%)$, patients with treatment failure $(4 / 35 ; 11.43 \%)$, defaulter $(4 / 35 ; 11.43 \%)$, and newly diagnosed drug-resistant TB patients $(5 / 35$; $14.29 \%$ ). MODS culture was positive in 19/35 patients; 16/19 (84.21\%) were smear-positive and 3/19 (15.79\%) were smear-negative patients. Sixteen patients did not have TB because of negative MODS culture result until the end of observation. The distribution of AFB smear positivity for these patients was shown in Figure 1. The median time to 


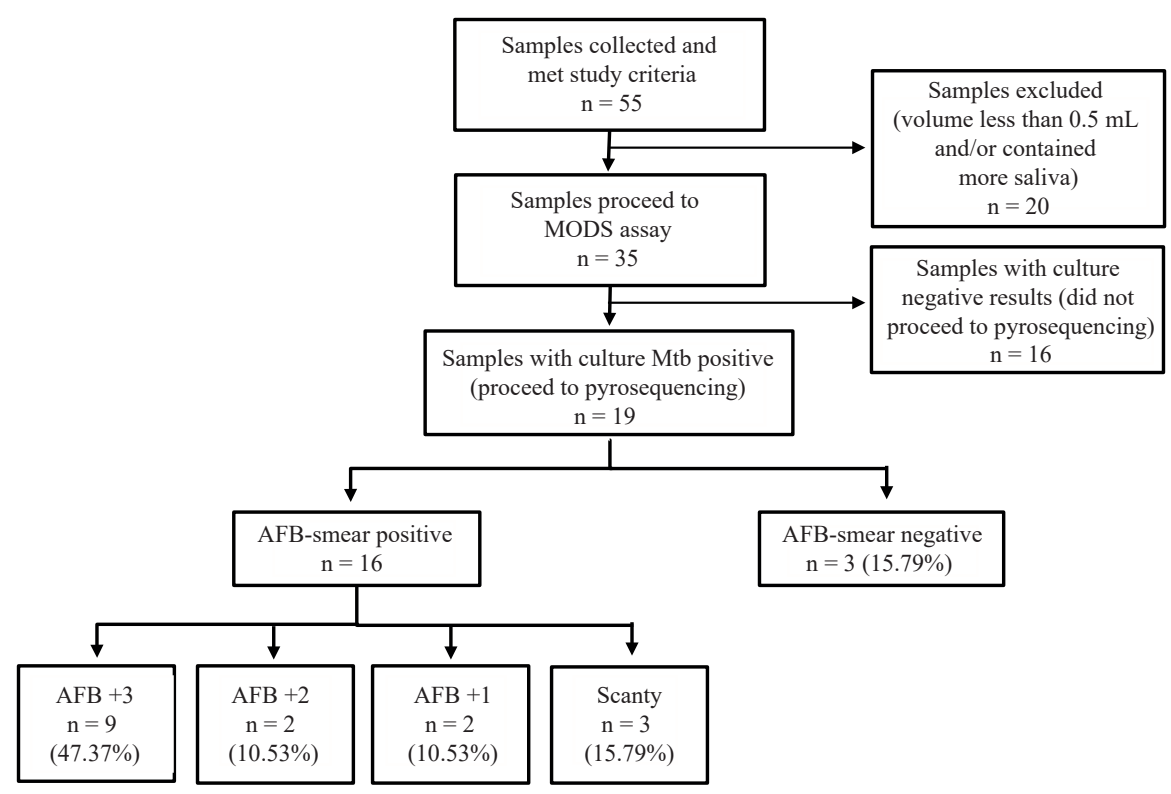

Figure 1. Distribution of AFB smear positivity in culture-positive samples.

culture positivity was 7 days (range 4-20 days). Nineteen culture-positive sputum specimens were further analyzed with PSQ. Specific sequence of IS6110 element (specific for $M$. tuberculosis complex) was identified in all specimens which confirmed the MODS results, yielded 100\% sensitivity of PSQ to detect the presence of $M$. tuberculosis in sputum (Table 1).

Results of drug susceptibility testing were available for isoniazid and rifampicin. Of 19 samples, 17 samples were phenotypically drug-resistant while 2 samples were

Table 1. Results of microscopic observation drug susceptibility (MODS) culture and pyrosequencing (PSQ) assay in identifying M. tuberculosis (Mtb).

\begin{tabular}{|c|c|c|c|c|}
\hline \multirow{2}{*}{$\begin{array}{l}\text { Number of } \\
\text { Sample }\end{array}$} & \multirow{2}{*}{$\begin{array}{c}\text { AFB* } \\
\text { grading }\end{array}$} & \multicolumn{2}{|c|}{ Culture Results } & \multirow[b]{2}{*}{ PSQ Results } \\
\hline & & Mtb & $\begin{array}{c}\text { Time to } \\
\text { Positivity }\end{array}$ & \\
\hline 1 & $(3+)$ & $+1+$ & 5 days & Positive \\
\hline 2 & $(3+)$ & $+1+$ & 6 days & Positive \\
\hline 3 & $(3+)$ & $+1+$ & 20 days & Positive \\
\hline 4 & $(3+)$ & $+1+$ & 13 days & Positive \\
\hline 5 & $(3+)$ & $+1+$ & 5 days & Positive \\
\hline 6 & $(3+)$ & $+1+$ & 4 days & Positive \\
\hline 7 & $(3+)$ & $+1+$ & 4 days & Positive \\
\hline 8 & $(3+)$ & $+1+$ & 4 days & Positive \\
\hline 9 & $(3+)$ & $+1+$ & 5 days & Positive \\
\hline 10 & $(2+)$ & $+1+$ & 7 days & Positive \\
\hline 11 & $(2+)$ & $+1+$ & 5 days & Positive \\
\hline 12 & $(1+)$ & $+1+$ & 5 days & Positive \\
\hline 13 & $(1+)$ & $+1+$ & 14 days & Positive \\
\hline 14 & $2 \mathrm{AFB} / 300 \mathrm{HPF}^{* *}$ & $+1+$ & 13 days & Positive \\
\hline 15 & $10 \mathrm{AFB} / 300 \mathrm{HPF}$ & $+1+$ & 20 days & Positive \\
\hline 16 & $4 \mathrm{AFB} / 100 \mathrm{HPF}$ & $+1+$ & 14 days & Positive \\
\hline 17 & Negative & $+1+$ & 13 days & Positive \\
\hline 18 & Negative & $+1+$ & 14 days & Positive \\
\hline 19 & Negative & $+1+$ & 14 days & Positive \\
\hline $20-35$ & Negative & $-1-$ & Negative & Not done \\
\hline
\end{tabular}

*Acid-fast bacilli; **high-power fields. 
drug-sensitive. PSQ assay could successfully generate complete resistance profiles in 16 (84.21\%) samples, of those $1(6.25 \%)$ was drug-sensitive, 2 (10.53\%) were rifampicin-resistance TB (RR-TB), 9 (56.25\%) were MDRTB, and $4(25 \%)$ were pre-extensively drug-resistant TB (preXDR-TB) (defined as MDR with additional resistance for fluoroquinolone or aminoglycoside). PSQ failed to amplify eis or gyrA for 3 samples. Among them, 1 was smear-negative and 2 had a low grade of smear results (scanty and 1+). Table 2 shows the distribution of mutations in each target in PSQ assay.

Sixteen of $19(84.21 \%)$ samples were observed as isoniazid-resistant by phenotypic DST. PSQ confirmed the results in 14 samples which mostly had mutations in katG. Two sputum samples showed no mutations in $k a t G$ or $i n h A$. Therefore, sensitivity of PSQ for predicting isoniazid resistance was $87.5 \%$ (14/16 specimens), and specificity was $100 \%$ as shown in Table 3 . For rifampicin, $17 / 19(89.47 \%)$ samples were observed as phenotypically resistant. Mutations were found in $\mathrm{rpoB}$ hotspot region in all samples by PSQ. One sample with phenotypically rifampicin-sensitive was found as resistant by PSQ, and this result was confirmed by Xpert MTB/RIF. Therefore, sensitivity of PSQ for rifampicin resistance was $100 \%$ and specificity was $50 \%$ (1/2 specimens) as shown in Table 4.

\section{Discussion}

Diagnosing TB is more challenging when the mycobacterial load is low, which can lead to smear-negative result.(19-21)
PSQ is a suitable rapid molecular test for the identification of M. tuberculosis complex as well as for rapid prediction of drug resistance directly from sputum. We showed that smear-negative/culture-positive patients could be detected by PSQ in this study (Table 1), indicating that sensitivity of PSQ for detecting TB is higher than AFB smear microscopy and comparable to culture method. Furthermore, the results could be obtained within 1-2 days after sputum collection, as opposed to 4-20 days needed to determine culture positivity using MODS assay. Higher smear positivity was associated with earlier growth of $M$. tuberculosis due to higher bacterial load (Table 1).

To date, studies of PSQ utilization are mostly performed on $M$. tuberculosis isolates. Only a few studies applied PSQ directly to sputum specimens. Studies from China and India reported that resistance profiles generated by PSQ were highly concordance with drug susceptibility results in all sputum samples.(22,23) However, these studies used smear-positive samples only. Another study in California reported a good accuracy of PSQ from clinical samples with different smear positivity, which included smear-negative patients (100\% detection rate).(15) Those studies showed a similar performance of PSQ with our study, therefore confirmed the suitability of PSQ for clinical specimens.

Some discrepancies between phenotypic and PSQ results were observed for both rifampicin and isoniazid. There were 2 isolates with isoniazid resistance but were sensitive by PSQ as no mutations were detected in katG and inhA promoter. This possibly caused by mutation(s) occured in other genes associated with isoniazid resistance,

Table 2. Resistance profile of $M$. tuberculosis by pyrosequencing.

\begin{tabular}{|c|c|c|c|}
\hline \multirow{2}{*}{$\begin{array}{c}\text { Drug and Its } \\
\text { Resistance Gene(s) }\end{array}$} & \multicolumn{2}{|c|}{ Frequency of Mutations Identified } & \multirow{2}{*}{ Information } \\
\hline & $\mathbf{n}$ & $\%(n=19)$ & \\
\hline \multicolumn{4}{|l|}{ Isoniazid } \\
\hline$k a t G$ & 9 & $47.37 \%$ & \\
\hline $\operatorname{inh} A$ & 5 & $26.32 \%$ & \\
\hline not found in both genes & 5 & $26.32 \%$ & \\
\hline \multicolumn{4}{|l|}{ Rifampicin } \\
\hline rрoB1 & 3 & $15.79 \%$ & \\
\hline гров2 & 16 & $84.21 \%$ & Novel mutation at codon $526 \mathrm{CTG} / \mathrm{CTC}$ in 1 sample \\
\hline not found in $r p o B$ & 1 & $5.26 \%$ & \\
\hline \multicolumn{4}{|l|}{ Floroquinolone } \\
\hline gyrA & 2 & $10.53 \%$ & \\
\hline not found in gyrA & 15 & $78.95 \%$ & \\
\hline \multicolumn{4}{|l|}{ Second-line injectable drugs } \\
\hline$r r s$ & 5 & $26.32 \%$ & Novel mutation at codon 1402 ATG/AAG in 2 samples \\
\hline eis & 0 & $0 \%$ & \\
\hline not found in both genes & 13 & $68.42 \%$ & \\
\hline
\end{tabular}


Table 3. Performance of pyrosequencing compared to gold standard microscopic observation drug susceptibility (MODS) drug susceptibility testing for isoniazid.

\begin{tabular}{|c|c|c|c|}
\hline \multirow[t]{2}{*}{ Pyrosequencing Assay } & \multicolumn{2}{|c|}{$\begin{array}{l}\text { Drug Susceptibility Testing } \\
\text { (Isoniazid) }\end{array}$} & \multirow[t]{2}{*}{ Total } \\
\hline & Resistant & Sensitive & \\
\hline Resistant & $14(73.68 \%)$ & 0 & $14(73.68 \%)$ \\
\hline Sensitive & $2(10.53 \%)$ & $3(15.79 \%)$ & $5(26.32 \%)$ \\
\hline Total & $16(84.21 \%)$ & $3(15.79 \%)$ & 19 \\
\hline
\end{tabular}

such as aphC-oxyR, efpA, fadE24, iniABC, kasA, nat, $n d h$, and $\operatorname{srmR}$.(24) PSQ for those samples also failed to generate a complete resistance profile. We observed that the samples belong to smear-negative and scanty samples. The low bacterial load may cause uneven distribution of $M$. tuberculosis DNA, resulting in a failure in several targets.

Another discrepancy was found for rifampicin in one sample which was sensitive by the phenotypic test but had a mutation in $r p o B$. This was a false-sensitive result since the mutation was at codon $511 \mathrm{CTG} / \mathrm{CCG}$ which is reported as disputed mutation and linked to low-level rifampicinresistance (below the minimum inhibitory concentration used in our phenotypic DST).(25,26) Disputed mutations are commonly found for rifampicin, made up over 11-13\% of all rроB mutations among failure and relapse cases. (25) Some studies suggested that rifampicin phenotypicmolecular discordance may be associated with poor outcomes among patients treated with recommended firstline regimen.(27,28)

We found two novel mutations in our samples. One mutation in rrs1402 ATG/AAG was suggestive for aminoglycoside resistance, but it has never been reported elsewhere. Its phenotypic test showed a sensitive result. This kind of novel mutations needs further assessment because it is commonly associated with a low-level and false-sensitive result. Another novel mutation was rpoB526 $\mathrm{CTG} / \mathrm{CTC}$ found in one sample, in which the phenotypic test confirmed the patient as rifampicin-resistant. Molecular
DST like PSQ could be used to detect disputed and novel mutations that will be missed by phenotypic test.

We showed that PSQ is a useful tool for predicting TB drug resistance rapidly which may help in shorten diagnostic process before phenotypic results available. This method can be performed from sputum specimens with varied bacterial load, even in low mycobacterial load as shown by smear microscopy results. This entire process can be done in a day, which is more expeditious compared to culture-based tests that could take days to weeks to obtain the results. The multi-loci feature of PSQ is suitable for clinical or public health settings requiring different testing conditions for different loci in the detection of multiple drug resistance. The ability for rapid and simultaneous detection of multidrug resistance is important to determine the appropriate treatment strategies for drug resistance patients. The administration of inappropriate drugs can contribute to poor outcomes and amplification of resistance patterns of M. tuberculosis to antituberculosis drugs.4-6

However, for a routine use of PSQ in clinical practice, previous experience and a proper skill in moleculartechniques are needed. Interpretation of novel mutations need further investigation. Furthermore, a report of mutations and their corresponding resistance should be made in a more userfriendly format for easier interpretation by the clinicians. Future studies are also needed to address the accuracy of this technique compared with current diagnostic tests such as GeneXpert, culture, and whole-genome sequencing.

Table 4. Performance of pyrosequencing compared to gold standard microscopic observation drug susceptibility (MODS) drug susceptibility testing for rifampicin.

\begin{tabular}{|c|c|c|c|}
\hline \multirow[t]{2}{*}{ Pyrosequencing Assay } & \multicolumn{2}{|c|}{$\begin{array}{c}\text { Drug Susceptibility Testing } \\
\text { (Rifampicin) }\end{array}$} & \multirow[t]{2}{*}{ Total } \\
\hline & Resistant & Sensitive & \\
\hline Resistant & $17(89.47 \%)$ & $1(5.26 \%)$ & $18(94.74 \%)$ \\
\hline Sensitive & 0 & $1(5.26 \%)$ & $1(5.26 \%)$ \\
\hline Total & $17(89.47 \%)$ & $2(10.53 \%)$ & 19 \\
\hline
\end{tabular}




\section{Conclusion}

Our study demonstrates high positivity rates of pyrosequencing to detect drug-resistant TB directly from sputum samples with different grades of smear microscopy, as the surrogate of bacterial load. The entire process of PSQ can be done in a day, which significantly shortens the turnaround time for obtaining the diagnosis. The assay can be used as a first prediction test of drug resistance prior to confirmation by phenotypic tests.

\section{References}

1. WHO. TB disease burden. In: Global Tuberculosis Report 2018. Geneva: WHO; 2018. p.27-66.

2. Nguyen L. Antibiotic resistance mechanisms in M. tuberculosis: an update. Archives of Toxicology. 2016; 90: 1585-604.

3. WHO. Diagnosis and treatment: TB, HIV-associated TB and drugresistant TB. In: Global Tuberculosis Report 2018. Geneva: WHO; 2018. p.67-102.

4. Azadi D, Motallebirad T, Ghaffari K, Shojaei H. Mycobacteriosis and tuberculosis: laboratory diagnosis. Open Microbiol J. 2018; 12: 41-58.

5. Dookie N, Rambaran S, Padayatchi N, Mahomed S, Naidoo K. Evolution of drug resistance in Mycobacterium tuberculosis: a review on the molecular determinants of resistance and implications for personalized care. J Antimicrob Chemother. 2018; 73: 1138-51.

6. Koch A, Cox H, Mizrahi V. Drug-resistant tuberculosis: challenges and opportunities for diagnosis and treatment. Curr Opin Pharmacol. 2018; 42: 7-15.

7. Al-Mutairi N, Ahmad S, Mokaddas E, Eldeen H, Joseph S. Occurrence of disputed rpoB mutations among Mycobacterium tuberculosis isolates phenotypically susceptible to rifampicin in a country with a low incidence of multidrug-resistant tuberculosis. BMC Infect Dis. 2019; 19: 3. doi: 10.1186/s12879-018-3638-z.

8. Agrawal M, Bajaj A, Bhatia V, Dutt S. Comparative study of GeneXpert with ZN stain and culture in samples of suspected pulmonary tuberculosis. J Clin Diagn Res. 2016; 10: DC09-12.

9. Oommen S, Banaji N. Laboratory diagnosis of tuberculosis: Advances in technology and drug susceptibility testing. Indian J Med Microbiol. 2017; 35: 323-31.

10. Govindaswamy A, Sakthi D, Pai R, Jeyaseelan L, Michael J. Pyrosequencing: a rapid and effective sequencing method to diagnose drug-resistant tuberculosis. J Med Microbiol. 2018; 67: 1212-6.

11. Torres J, Paul L, Rodwell T, Victor T, Amallraja A, Elghraoui A, et al. Novel katG mutations causing isoniazid resistance in clinical M. tuberculosis isolates. Emerg Microbes Infect. 2015; 4: e42. doi: 10.1038/emi.2015.42.

12. Sheen P, Requena D, Gushiken E, Gilman R, Antiparra R, Lucero B, et al. A multiple genome analysis of Mycobacterium tuberculosis reveals specific novel genes and mutations associated with pyrazinamide resistance. BMC Genomics. 2017; 18: 769. doi: 10.1186/s12864-017-4146-z.

13. Zhang S, Chen J, Cui P, Shi W, Zhang W, Zhang Y. Identification of novel mutations associated with clofazimine resistance in Mycobacterium tuberculosis. J Antimicrob Chemother. 2015; 70:
2507-10.

14. Lyu J, Zhang J, Ren X. Detection and identification of bacterial pathogens directly from sputum samples by pyrosequencing. J Med Microbiol. 2019; 68: 368-73.

15. Lin S, Rodwell T, Victor T, Rider E, Pham L, Catanzaro A, et al. Pyrosequencing for rapid detection of extensively drug-resistant mycobacterium tuberculosis in clinical isolates and clinical specimens. J Clin Microbiol. 2013; 52: 475-82.

16. Nimmo C, Shaw L, Doyle R, Williams R, Brien K, Burgess C, et $a l$. Whole genome sequencing Mycobacterium tuberculosis directly from sputum identifies more genetic diversity than sequencing from culture. BMC Genomics. 2019; 20: 389. doi: 0.1186/s12864-019$5782-2$.

17. Kementerian Kesehatan RI. Peraturan Menteri Kesehatan Nomor 67 Tahun 2016 tentang Penanggulangan Tuberkulosis. Jakarta: Kementerian Kesehatan RI; 2016.

18. Moore D. MODS - a user guide. Lima: Universidad Peruana Cayetano Heredia; 2006.

19. Zhang Z, Sng L, Yong Y, Lin L, Cheng T, Seong N, et al. Delays in diagnosis and treatment of pulmonary tuberculosis in AFB smearnegative patients with pneumonia. Int J Tuberc Lung Dis. 2017; 21 : 544-9.

20. Reviono R, Setianingsih W, Damayanti K, Ekasari R. The dynamic of tuberculosis case finding in the era of the public-private mix strategy for tuberculosis control in Central Java, Indonesia. Glob Health Action. 2017; 10:1353777. doi: 10.1080/16549716.2017.1353777.

21. Rasool G, Khan A, Mohy-Ud-Din R, Riaz M. Detection of Mycobacterium tuberculosis in AFB smear-negative sputum specimens through MTB culture and GeneXpert ${ }^{\circledR}$ MTB/RIF assay. Int J Immunopathol Pharmacol. 2019; 33: 2058738419827174. doi: 10.1177/2058738419827174.

22. Zheng R, Zhu C, Guo Q, Qin L, Wang J, Lu J, et al. Pyrosequencing for rapid detection of Tuberculosis resistance in clinical isolates and Sputum samples from re-treatment Pulmonary Tuberculosis patients. BMC Infect Dis. 2014; 14: 200. doi: 10.1186/1471-233414-200.

23. Nambiar R, Shah D, Ajbani K, Kazi M, Sadani M, Shetty A, et al. Evaluation of pyrosequencing for extensive drug resistance-defining anti-tuberculosis drugs for use in public healthcare. Tuberculosis. 2018; 110: 86-90.

24. Seifert M, Catanzaro D, Catanzaro A, Rodwell T. Genetic mutations associated with isoniazid resistance in mycobacterium tuberculosis: a systematic review. PLOS ONE. 2015; 10: e0119628. doi: 10.1371/ journal.pone.0119628.

25. Chiang T, Fan S, Jou R. Performance of an Xpert-based diagnostic algorithm for the rapid detection of drug-resistant tuberculosis among high-risk populations in a low-incidence setting. PLOS ONE. 2018; 13: e0200755. doi: 10.1371/journal.pone.0200755.

26. Ocheretina O, Shen L, Escuyer V, Mabou M, Royal-Mardi G, Collins $\mathrm{S}$, et al. Whole genome sequencing investigation of a tuberculosis outbreak in Port-au-Prince, Haiti caused by a strain with a "lowlevel" rpoB mutation L511P - insights into a mechanism of resistance escalation. PLOS ONE. 2015; 10: e0129207. doi: 10.1371/journal.pone.0129207.

27. Van Deun A, Aung K, Hossain A, de Rijk P, Gumusboga M, Rigouts $\mathrm{L}$, et al. Disputed rpo B mutations can frequently cause important rifampicin resistance among new tuberculosis patients. Int J Tuberc Lung Dis. 2015; 19: 185-90.

28. Van Deun A, Decroo T, Kya Jai Maug A, Hossain M, Gumusboga M, Mulders $\mathrm{W}$, et al. The perceived impact of isoniazid resistance on outcome of first-line rifampicin-throughout regimens is largely due to missed rifampicin resistance. PLOS ONE. 2020; 15: e0233500. doi: 10.1371/journal.pone. 0233500 . 